\title{
Theory Research on the Solar Cell Martial of GaN by a Novel Method
}

\author{
Fengqi Quan \\ State Grid Liaoning Electric Power Development Co. \\ Ltd, \\ Shenyang, China, \\ e-mail: qfqsy@163.com \\ Hongyu Zhang \\ State Grid Liaoning Electric Power Development Co. \\ Ltd, \\ Shenyang, China, \\ e-mail: zhysy@126.com

\section{Peiyuan Cong} \\ State Grid Liaoning Electric Power Development Co. \\ Ltd, \\ Shenyang, China, \\ e-mail: cpysy@126.com
}

\author{
Haijun $\mathrm{Yu}$ \\ State Grid Liaoning Electric Power Development Co. \\ Ltd, \\ Shenyang, China, \\ e-mail: yhjsy@163.com \\ Hao Huang \\ State Grid Liaoning Electric Power Development Co. \\ Ltd, \\ Shenyang, China, \\ e-mail: hhsy@ 126.com
}

devices frequency microwave devices application has broad prospects.

Indium nitride $(\mathrm{InN})$ is probably the most important semiconductor since silicon and Gallium nitride $(\mathrm{GaN})$. By virtue of its wide band gap and some other excellent properties such as high breakdown voltage, high saturated drift velocity, high mechanical and thermal stability; it has potential for a wide range of solid-state optoelectronic and electronic applications. Actually, high quality GaN films have already been exploited commercially for solar cells, and the $\mathrm{GaN} / \mathrm{InGaN}$ solar cells with internal quantum efficiencies as high as $60 \%$ were also fabricated on sapphire substrates using MOCVD method ${ }^{[6]}$. Therefore, more and more efforts are made to efficiently grow $\mathrm{GaN}$ films of better quality due to the practical application.

At present, the deposition of GaN films have been reported by using different methods, such as metal organic vapor-phase epitaxy (MOVPE), molecular beam epitaxy (MBE) and hydride vapor-phase epitaxy (HVPE), Pulsed laser deposition (PLD) as well as sputtering. However, the grown of high quality GaN films is limited to the small deposition temperature. This is mainly because of the decomposition of the $\mathrm{GaN}$ films under the high temperature. It is worthwhile to mention here that there have been limited reports on high quality $\mathrm{GaN}$ films deposited at low temperature. In this study, the high quality InN films are successfully achieved at the low temperature by electron cyclotron resonance plasma enhanced metal organic chemical vapour deposition (ECR-PEMOCVD) system. The ECR-PEMOCVD system combines advanced features of MBE and MOCVD, which is a new type of as-grown film deposition technology, and this method can produce high-density charge and 
stimulated particles by using microwave electron cyclotron resonance discharge at low pressure. Since the multicusp cavity-coupling ECR plasma source was adopted to provide active precursors, the growth temperatures were effectively decreased and the $\mathrm{N}_{2}$ reactivity can be remarkably enhanced by the ECR process, this method was necessary for the formation of $\mathrm{GaN}$ films under the low temperature.

GaN material series having a low heat generating rate and a high breakdown field, high-power electronic devices is the development of high-temperature and highfrequency microwave devices the important material. Now, with advances in technology breakthroughs MBE GaN material applications and critical film growth techniques, successfully grown a variety of $\mathrm{GaN}$ heterostructures. GaN material prepared by a metal field effect transistor (MESFET), heterojunction field effect transistor (HFET), modulation doped field effect transistor (MODFET) and other new devices. The modulationdoped AlGaN / GaN structure with high electron mobility $(2000 \mathrm{~cm} 2 / \mathrm{v} \cdot \mathrm{s})$, a high saturation velocity $(1 \times 107 \mathrm{~cm}$ /s), a lower dielectric constant, is the production of microwave devices priority materials; $\mathrm{GaN}$ wide band gap $(3.4 \mathrm{eV})$ and sapphire and other materials as the substrate, thermal performance, is conducive to the device operating at high power conditions.

In this study, GaN films were prepared by ECRPEMOCVD system at the low temperature for different TMIn fluxes. The results demonstrate that the dense and uniform $\mathrm{GaN}$ films with highly c-axis preferred orientation are successfully achieved on the sapphire substrates under optimized TMIn flux, and the RHEED and XRD results of the optimized GaN film show the high quality with the high c-orientation along the substrates. The AFM images show that the uniform and dense $\mathrm{GaN}$ is of the smooth surface. The obtained GaN/Sapphire structure will be used as a wide range of high-power semiconductor devices and the solar cells.

Three samples of $\mathrm{GaN}$ films with different $\mathrm{SiNx}$ depositing time were grown on Si-terminal (0001) 6H-SiC substrates (n-type) using MOCVD in a Thomas Swan $3 \times 2$ close-coupled showerhead reactor. The substrates used here were commercial wafers with on-axis direction. The samples were first ramped to $1100{ }^{\circ} \mathrm{C}$ for hydrogen $(\mathrm{H} 2)$ baking, to remove the surface damage induced by mechanical polish. Then an AlGaN buffer layer was deposited at $1040{ }^{\circ} \mathrm{C}$ : TMGa and TMAl flow kept on $21.99 \mu \mathrm{mol} / \mathrm{min}$ and $4.68 \mu \mathrm{mol} / \mathrm{min}$, respectively, while NH3 flow was $3 \mathrm{slm}$. Then a $1 \mu \mathrm{m} \mathrm{GaN} \mathrm{template} \mathrm{was}$ prepared at the same temperature. In this time, a $6 \mathrm{~nm}$ SiNx nano-mask was induced by the same temperature, After SiNx time, a $4 \mu \mathrm{m}$ GaN were sequentially grown with the same condition as the former GaN. The highresolution X-ray diffraction (XRD), Photoluminescence (PL), Raman, scanning electron microscopy (SEM) is carried out to characterize the quality of GaN flims.

\section{EXPERIMENTS}

In this study, the samples were prepared on sapphire substrates using an electron cyclotron resonance plasma enhanced metal organic chemical vapour deposition (ECR-PEMOCVD) system at various TMIn fluxes. The experimental device of ECR-PEMOCVD is a new type of thin film deposition technology, because this technology combines advanced features of MBE with MOCVD and uses microwave electron cyclotron resonance discharge at low pressure to produce high-density charge and stimulated particles. In this case, the gas is very easy to achieve chemical reaction and then deposit the films. So compared to the conventional plasma enhanced chemical deposition (PECVD), the ECR plasma conditions has more advantages.

First, the substrate will be using an ultrasonic cleaning in acetone for 5 minutes, then for 10 minutes using an ultrasonic washing in ethanol, and finally with demonized water, ultrasonically cleaned for 12 minutes and finally dried with nitrogen.

Prior to the growth, the substrate surface was thermally cleaned by pure $\mathrm{H}_{2}$ at $600^{\circ} \mathrm{C}$ for $20 \mathrm{~min}$ with the $\mathrm{H}_{2}$ flux of $50 \mathrm{sccm}$ added $10 \mathrm{sccm} \mathrm{N}_{2}$. The next process is the surface of sapphire substrate was nitridation using the plasma with the $\mathrm{N}_{2}$ flux of at $450{ }^{\circ} \mathrm{C}$ for $5 \mathrm{~min}$. The purpose of the nitridation procedure are to form $\mathrm{AlN}$ buffer layer, which can help lowering the mismatch between sapphire and $\mathrm{InN}$ down to $12 \%$, and the ways are dramatically improving the surface morphology and crystalline quality of GaN film.

\section{RESULTS AND DISCUSSION}

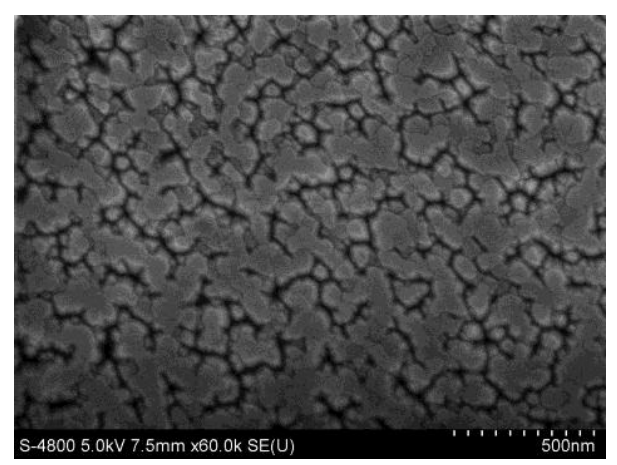

Figure SEM image of GaN surface after 20 min SiNx deposition

Fig .1 provides the SEM image of GaN surface after 20 min SiNx growth. It can be seen obviously that the grown SiNx covers the surface partially, making a porous structure. The purpose of taking a long SiNx growth time (20 $\mathrm{min}$ ) is to clearly show the porous structure.

The FWHM of three samples as a function of $\mathrm{SiNx}$ interlayer time is shown in Fig .2. The full width at half maximum (FWHM) of (002) diffraction is correlated with the density of screw dislocations, while asymmetric (102) is sensitive to the pure edge and mixed dislocations [4]. The TD density can be calculated by the equation [5]. 
Where $\rho$ s is the screw dislocation density, $\rho$ e is the edge dislocation density, $\beta(002)$ and $\beta(102)$ are the full width at half maximum (FWHM) of (002) and (102) diffraction, while Bs and Be are Burger vector sizes of the screw $(c=0.5185 \mathrm{~nm})$ and edge $(\mathrm{a}=0.3188 \mathrm{~nm})$ components. The screw dislocation densities are calculated as $7.9 * 107 \mathrm{~cm}-2,4.2 * 107 \mathrm{~cm}-2$ for two samples, and the edge dislocation densities are 5.7*108 $\mathrm{cm}-2$ and $1.7 * 108 \mathrm{~cm}-2$. There are two mechanisms responsible for the TD termination. First, the nano-SiNx mask is preferentially formed at the TD cores because of the presence of $\mathrm{N}$-dangling bonds for $\mathrm{Si}-\mathrm{N}$ formation to terminate TD [6]. Second, due to Fig .1, the in situ SiNx interlayer incompletely covers the underlying $\mathrm{GaN}$ template whicn acts as a nano-scale mask. Then the subsequent growth is similar to ELO [7], lots of TDs were terminated, and the quality of $\mathrm{GaN}$ was improved.

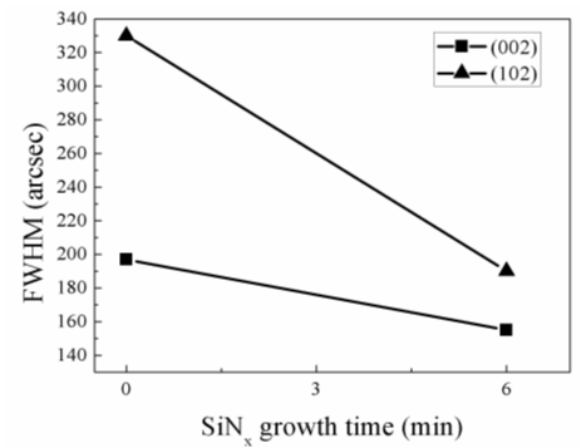

Figure 2. Relationship between SiNx time and FWHM of (002) and (102) diffraction

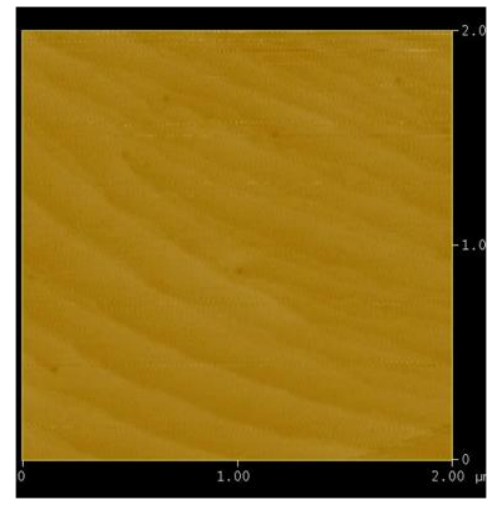

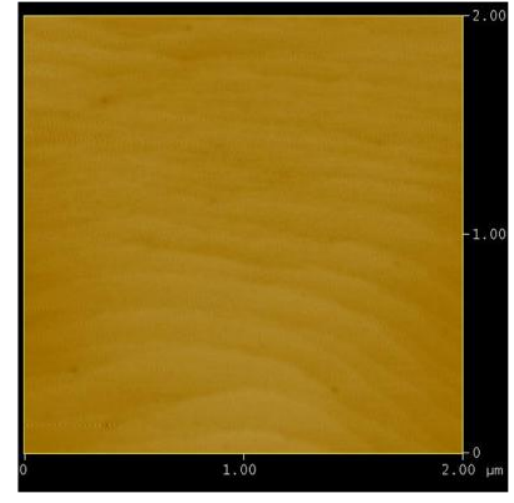

Figure 3. Atomic force microscopy of twosamples

Fig .3 shows the AFM images of the samples. Step terminations combined with some dark dots can be clearly observed. The step terminations indicate that a step-flow mode is formed in the samples. The dark dots in the surfaces are related to edge dislocations or mix dislocations [8]. The RMSs are 0.25 and $0.17 \mathrm{~nm}$ respectively. The surface of $\mathrm{SiNx}$ sample is smoother than the conventional one.

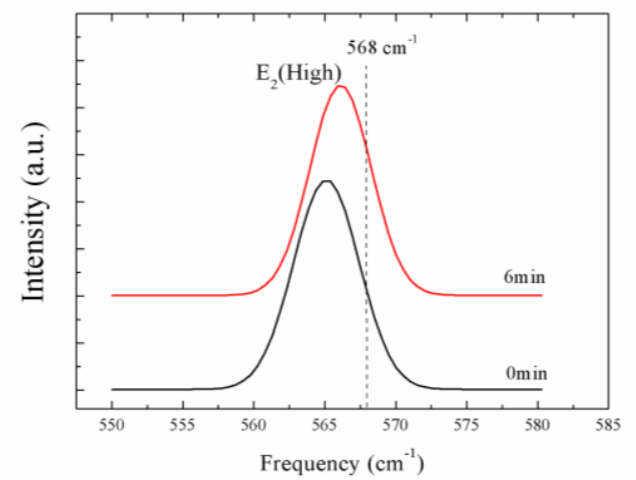

Figure 4. The room temperature of Raman spectra of two samples

Fig .4 displays the Raman spectra near E2 mode. In the Raman scattering geometry, the GaN E2 photon peak is a convenient indicator of strain, however, a tensile stress will result in a photon frequency redshift while a compress stress must be responsible for a photon frequency blueshift. The E2 phonon lines of GaN layers in this study all subjected to a low-frequency shift is indicative of the existence of a biaxial tensile strain in GaN layers.

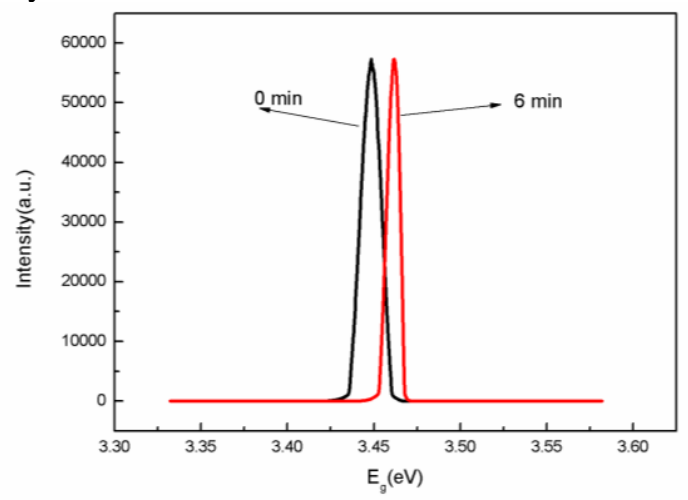

Figure 5. Low temperature PL of two samples at 10K, 
Fig .5 shows the near-band edge low temperature PL spectra of samples at $10 \mathrm{~K}$. The dominant line emission in these spectra is related to recombination of donor bound excitons (D0X). As SiNx interlayer induced, there is an obvious blueshift of D0X peaks. It is well know that the residual stress can affect the energy band gap and a tensile stress will result in a decrease of energy band gap while a compress stress cause an increase of band gap. The two samples subject to a lower photon energy compared to $3.472 \mathrm{eV}$ of a strain-free bulk GaN [9]. Thus, the samples in this study suffered tensile strain. The FWHM of standard process was $10.5 \mathrm{meV}$, and rapidly decrease to5.1 meV with a $6 \mathrm{~min}$ nano-SiNx interlayer. This decrease indicates the $\mathrm{SiNx}$ interlayer considerably improved the optical properties of GaN[10].

\section{CONCLUSION}

In the letter, we employ an in situ SiNx as mask during $\mathrm{GaN}$ process to promote lateral growth. TDs were terminated effectively by bending to the facets of $\mathrm{GaN}$ islands, or annihilated by reacting with each other of opposite Burgers vector, while nano-SiNx mask preferentially forming at the TD cores may be another mechanism responsible for the TD termination. Also, the optical quality is improved with the increasing $\mathrm{SiNx}$ time. Finally, the improvement of quality and strain relaxation of GaN epilayer is achieved by the in situ SiNx interlayer.

\section{ACKNOWLEDGMENT}

The authors gratefully acknowledge the contribution of co-workers and reviewers' comments.

\section{REFERENCES}

[1] H. M. Ng, D. Doppalapudi, and T. D. Moustakas, "The role of dislocation scattering in n-type GaN films," APPL. PHYS. LETT., vol.73,pp.821-823,May 1998

[2] F. Yun, Y. T. Moon, and Y. Zhu, "Efficacy of single and double SiNx interlayers on defect reduction in $\mathrm{GaN}$ overlayers grown by organometallic vapor-phase epitaxy," J. Appl. Phys., vol.7,pp.35-39,May 2005

[3] X. L. Fang, Y. Q. Wang, and H. Meidia, "Reduction of threading dislocations in GaN layers using in situ deposited silicon nitride masks on AlN and GaN nucleation layer," Appl. Phys. Lett. 84 vol.84,.pp.484-487,July 2004

[4] A. E. Romanov and J. S. Speck, "Stress relaxation in mismatched layers due to threading dislocation inclination," APPL. PHYS. LETT., vol.83,.pp. 2569-2571,July 2003

[5] M. Takeuchi, H. Shimizu, and R. Kajitani, "Al- and N-polar AlN layers grown on $c$-plane sapphire substrates by modified flowmodulation MOCVD," J. Cryst. Growth., vol.8,.pp.360-365,May 2007

[6] S. Tanaka, M. Takeuchi, and Y. Aoyagi, "Anti-Surfactant in IIINitride Epitaxy -Quantum Dot Formation and Dislocation Termination,” Jpn. J. Appl. Phys., vol.39,.pp.381-384,March 2000

[7] M. J. Kappers, R. Datta, and R. A. Oliver, "Threading dislocation reduction in (0 001$) \mathrm{GaN}$ thin films using $\mathrm{SiN}_{x}$ interlayer," J. Cryst. Growth., vol.35,.pp. 70-74,June 2007

[8] T.Hino,S.Tomiya,T.Miyajima, and K.Yanashima, "Characterization of threading dislocations in GaN epitaxial layers,"Appl. Phys. Lett., vol.68,pp. 3421-3423,July 2000

[9] K. Pakula, A. Wysmolek, and K. P. Korona, "Luminescence and reflectivity in the exciton region of homoepitaxial GaN layers grown on GaN substrates," Solid State Commun., vol.23,.pp. 919922,May 1998

[10] X.L.Liu, F.W.Qin, and J.M.Bian, "The Preparation and Characteristics of InxGa1-xN $(0.06 \leq \mathrm{x} \leq 0.58)$ Films Deposited by ECR-PEMOCVD,"CHIN. PHYS. LETT. vol.23,.pp. 108-112,May 2011 